\title{
ARTICLE OPEN \\ Nanoembryonic thermoelastic equilibrium and enhanced properties of defected pretransitional materials
}

\author{
Ye-Chuan $\mathrm{Xu}^{1}$, Wei-Feng Rao ${ }^{1}$, John W. Morris $\mathrm{Jr}^{2}$ and Armen G. Khachaturyan ${ }^{2,3}$
}

Behaviors of displacive phase-transforming materials above the temperature of transformation, where abnormal thermal, elastic, magnetic properties are often observed, are mostly explained by intrinsic peculiarities in electronic/atomic structure. Here, we show these properties may also be attributed to extrinsic effects caused by a thermoelastic equilibrium in highly defected pretransitional materials. We demonstrate that the stress concentration near stress-generating defects such as dislocations and coherent precipitates could result in the stress-induced transformation within nanoscale regions, producing equilibrium embryos of the product phase. These nanoembryos in thermoelastic equilibrium could anhysteretically change their equilibrium size in response to changes in applied stress or magnetic fields leading to superelasticity or supermagnetostriction. Similar response to cooling may explain the observed diffuse phase transformation, changes in the coefficient of thermal expansion and effective elastic modulus, which, in turn, may explain the invar and elinvar behaviors.

npj Computational Materials (2018)4:58; doi:10.1038/s41524-018-0114-7

\section{INTRODUCTION}

Large and low/nonhysteretic strain responses to externally applied stress, electric and/or magnetic fields are of great interests for sensors, actuators, and many other applications. However, the intrinsic anhysteretic strain response of thermodynamically stable and homogeneous materials is usually very small, e.g., $10^{-5}-10^{-4}$ under a magnetic field. The responses involving displacive, martensitic, ferroelectric, or ferroelastic transformations could be orders of magnitude higher. Hereafter, we call for brevity all these transformations as martensitic transformation (MT). For example, applying a magnetic field to the Fe-based alloys at room temperature could induce an fcc (paramagnetic) to bcc (ferromagnetic) martensitic transformation, ${ }^{1,2}$ resulting in a giant strain response that could be interpreted as a supermagnetostriction. Unfortunately, this response is highly hysteretic or even irrecoverable, and requires prohibitively high magnetic field. ${ }^{3,4} \mathrm{~A}$ major reason is that the large lattice mismatch between the product and parent phases, which is also the reason for the large response, causes a high strain energy barrier of nucleating the product phase.

The difficulty of nucleation could be overcome by introducing static stress-generating defects such as dislocations and/or coherent nanoprecipitates into a pretransitional state. Hereafter, the term pretransitional material describes a stable/metastable homogeneous phase with a latent instability with respect to a displacive, martensitic, ferroelectric, or ferroelastic transformation that develops upon cooling to (but always above) the starting temperature of martensitic transformation, $M_{s}$. The local stress induced by the defects in the pretransitional state could eliminate the nucleation barrier if the stress level in the stress concentration regions around defects reaches a critical level. ${ }^{5-7}$ This triggers a barrierless MT forming equilibrium nanosized martensitic embryos in the pretransitional material at $T>M_{s}$. This local equilibrium represents a thermoelastic equilibrium provided by the energy balance between the stress-induced defect-embryo interaction, which promotes the embryo formation, and chemical free energy together with a part of the strain energy, which suppresses it.

These embryos formed at $T>M_{s}$ are physically different from the fluctuation-assisted nuclei formed in the conventional heterogeneous nucleation at $T<M_{s}{ }^{8-10}$ In particular, the embryos at $T>M_{s}$ are always equilibrium coherent nanosized particles, while the conventional fluctuation-assisted nuclei at $T<M_{s}$ are nonequilibrium particles that equilibrate by growing until they reach macroscopic dimensions. The conventional thermoelastic equilibrium $^{11}$ is a known effect describing the two-phase equilibrium of coherent macroscopic crystals. They form below the $M_{s}$ and can be heated up above the $M_{s}{ }^{9}{ }^{9,10,12,13}$ We still use this term, however, to name a conceptually similar but different equilibrium describing a mixed state consisting of nanosized embryos above the $M_{s}$. This paper shows that a material in the nanoembryonic equilibrium may have anhysteretic giant responses to external stimuli such as superelasticity ${ }^{14}$ and supermagnetostriction, ${ }^{15,16}$ and the nanoembryonic mechanism could, at least partly, explain the existence of diffuse phase transformations (DPT), ${ }^{17}$ negative thermal expansion coefficients, elastic softening upon cooling and their particular cases, Invar ${ }^{18,19}$ and Elinvar ${ }^{18,19}$ effects.

In thermoelastic equilibrium at $T>M_{s}$, the sizes of nanosized embryos and their volume fraction, $\omega$, are internal thermodynamic parameters. Since the rate of a MT is usually much higher than that of continuously changing external stimuli, the evolution of embryos can be regarded as a quasi-static process passing along a line of equilibrium states. Therefore, the value $\omega$ is an unambiguous continuous function of external parameters such as temperature $T$, stress $\sigma$, and magnetic field $H$, i.e., $\omega=\omega(T, \sigma, H)$. The value of $\omega$ increases when $T$ approaches $M_{s}$ upon cooling and

\footnotetext{
${ }^{1}$ Department of Materials Physics, and IEMM, Nanjing University of Information Science and Technology, Nanjing 210044 , China; ${ }^{2}$ Department of Materials Science and Engineering, University of California, Berkeley, CA 94720, USA and ${ }^{3}$ Department of Materials Science and Engineering, Rutgers University, Piscataway, NJ 08854, USA Correspondence: W-F. Rao (wfrao@nuist.edu.cn)
}

Received: 11 March 2018 Revised: 26 September 2018 Accepted: 3 October 2018

Published online: 05 November 2018 


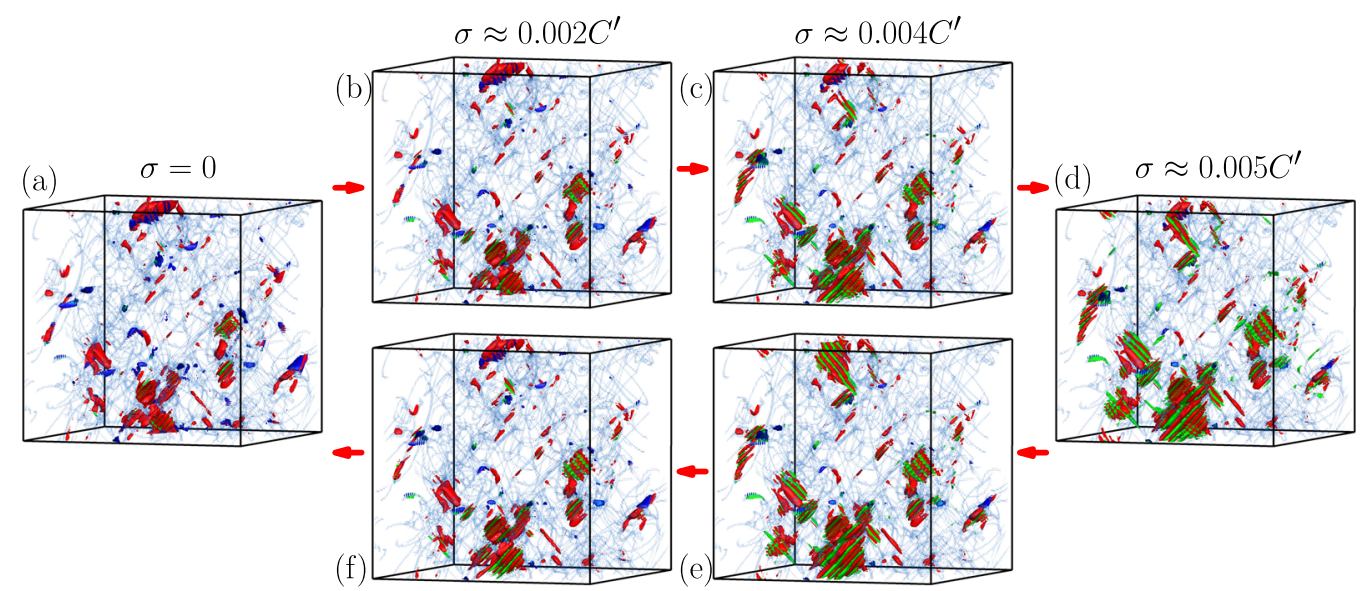

Fig. 1 The evolution of nanoembryonic martensitic structures in the dislocated sample with $\sim 5 \%$ plastic deformation (dislocation density $\sim 1 \times 10^{11} \mathrm{~cm}^{-2}$ ) caused by quasi-static application of external stress. The simulation is performed at T/M $\sim 1.15$. The static dislocation structure was generated by simulating the plastic deformation of defect-free austenite, which is visualized by the dislocation lines in cyan. Embryos of different orientation variants are colored in red, blue and green, respectively. The red arrows indicate the sequence of the structures obtained as the stress is cycled
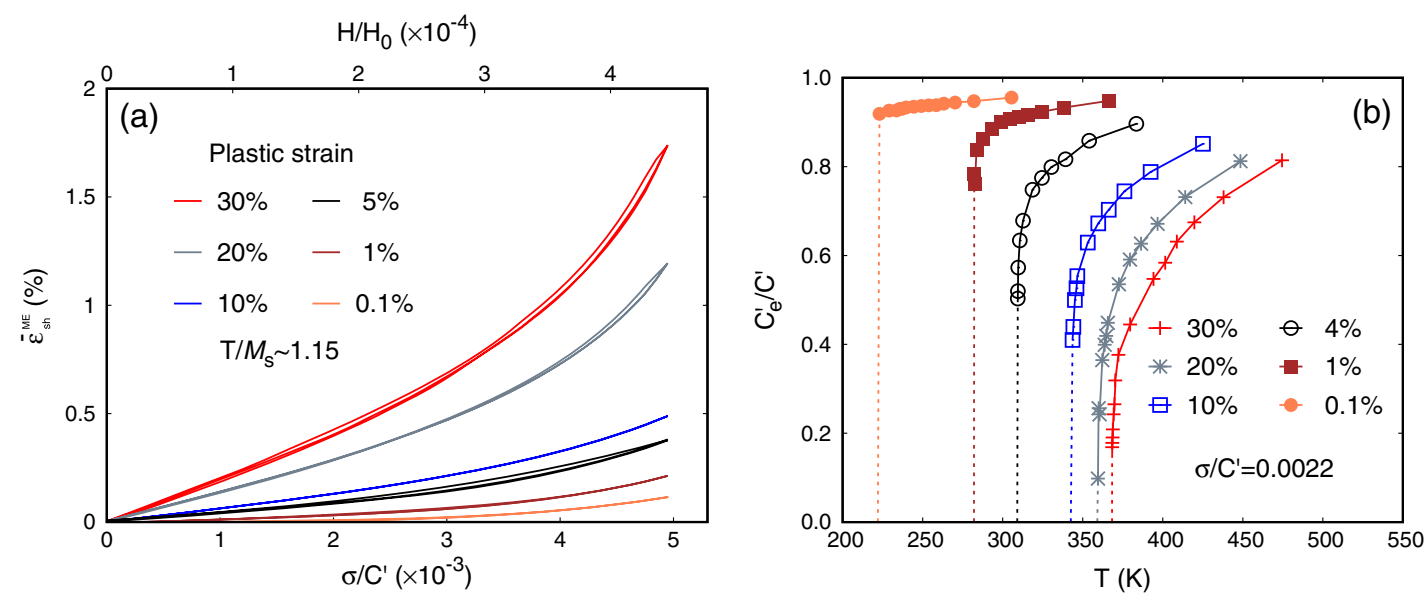

Fig. 2 a Simulated shear strain amplifications versus cycling applied stress and magnetic field ("magnetic stress") for samples with different plastic deformations at reduced temperature of $T / M_{s} \sim 1.15$. b Temperature dependence of the effective shear modulus for samples with different plastic deformation. The modulus was calculated at a constant shear stress level of $\sigma / C^{\prime}=0.0022$. The dashed lines indicate the temperatures at which the embryos detach from the defects and grow to consume the whole simulation box

undergoes a jump at $T=M_{s}$ reflecting the start of the macroscopic-scale MT. The continuity of $\omega$ was observed at $T>$ $M_{s}$ in ferroelectric pretransitional materials ${ }^{17}$ and was interpreted as a manifestation of DPT.

According to Eshelby theory, ${ }^{20}$ the evolution of nanosized embryos in finite crystals contributes to homogeneous strain, $\bar{\varepsilon}_{i j}$, that is proportional to the value of $\omega$, i.e., $\bar{\varepsilon}_{i j}=\varepsilon_{i j}^{0} \omega(T, \sigma, H)$, where $\varepsilon_{i j}^{0}$ is the eigenstrain describing the lattice misfit between the parent and product phases. Since $\varepsilon_{i j}^{0}$ is a Bain strain that is large for an $\mathrm{fcc} \rightarrow \mathrm{bcc}$ MT and $\omega(T, \sigma, H)$ is a single-valued function, the strain response to external stimuli, $\bar{\varepsilon}_{i j}$, can be giant and anhysteretic. In addition, the volumetric effect of $\varepsilon_{i j}^{0}$, the trace $\varepsilon_{i i}^{0}$, is always positive for an $\mathrm{fcc} \rightarrow \mathrm{bcc}$ MT. Therefore, the volume expansion of the system due to the growth of embryos upon cooling could provide a negative contribution to the thermal expansion coefficient, which may, at least, partially explain the still controversial origin of invar effect. ${ }^{18,19,21,22}$

\section{RESULTS}

We used 3D computer simulations of a pretransitional material with stress-generating defects at $T>M_{\mathrm{s}}$ to show the formation of an equilibrium mixed state consisting of nanoembryos, and to demonstrate the effects of shifting this equilibrium resulting in enhanced properties. For illustration, we considered a generic Fe$31 \mathrm{at} \% \mathrm{Ni}$ alloy with a set of reasonably fitted parameters (Supplemental materials). This alloy is probably one of the best studied martensitic material that is a pretransitional paramagnetic austenite at room temperature and ferromagnetic martensite at $T$ $<M_{s}$. We first simulated different distributions of dislocations or nanoprecipitates by prototyping either plastic deformation or precipitation (Supplemental materials). The obtained "samples" with defects, which are assumed to be static hereafter, were then brought to a temperature above the $M_{s}$ where the embryos become thermodynamically stable in the stress fields of defects (Fig. 1). We then studied how changes in temperature, applied stress and magnetic field affect the system. The applied shear stress was chosen as,

$$
\sigma_{i j}^{a p p}=\frac{\sigma}{2}\left[\begin{array}{ccc}
0 & 0 & 0 \\
0 & 1 & 0 \\
0 & 0 & -1
\end{array}\right],
$$

where the coordinate axes are taken parallel to the 

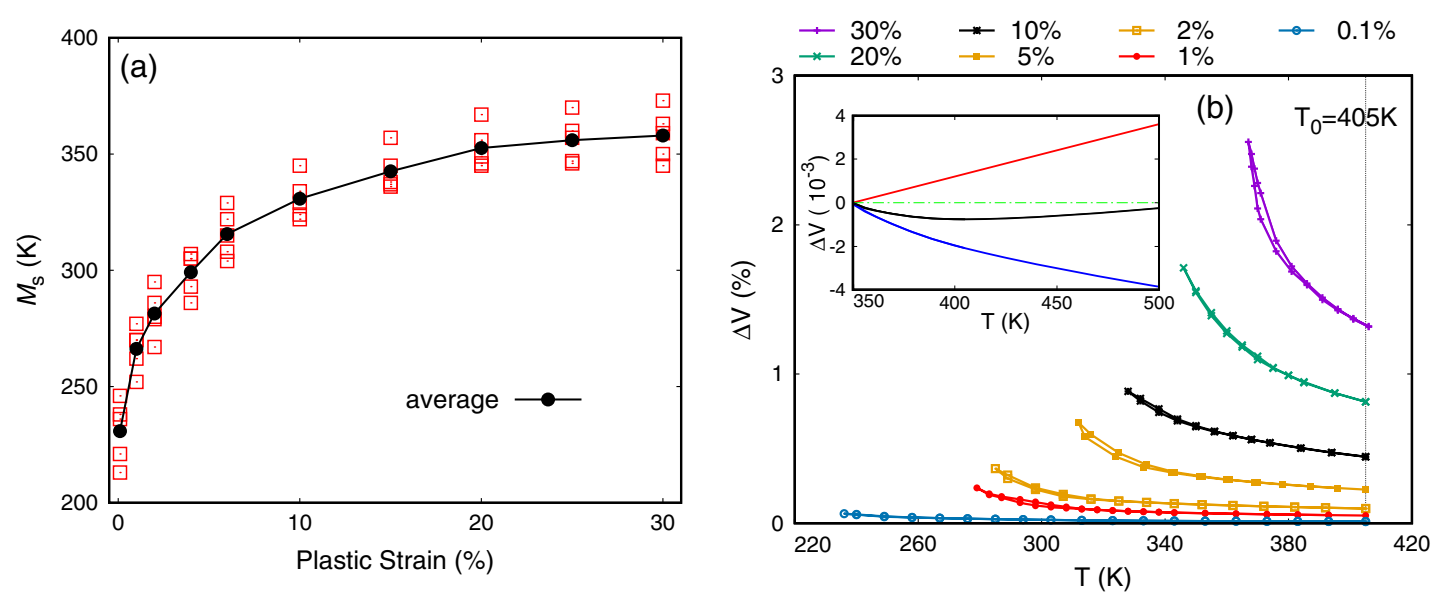

Fig. 3 a Simulated values of $M_{s}$ versus the dislocation-generating plastic strain, where data points show the $M_{s}$ measured for five sets of different dislocation configurations. The average values of $M_{s}$ are connected by segments to guide to the eye. $\mathbf{b}$ Simulated dependence of the volume change on temperature for samples with different plastic deformation. Inset: volume change versus temperature for an elastically isotropic alloy with a volumetric coefficient of thermal expansion of $a=24 \times 10^{-6} / \mathrm{K}^{19}$ (red line), simulated volume change versus temperature for a sample with $\sim 10 \%$ plastic strain (blue line), and the sum of both (black line)

directions of the fcc lattice. Figure 1 illustrates the change of embryo distribution caused by the dislocation structures under quasi-static cycling of $\sigma$. The embryos grow and shrink with $\sigma$, and their response to $\sigma$ is practically anhysteretic.

The increase of $\omega$ with $\sigma$ adds to the total macroscopic strain, $\bar{\varepsilon}_{i j}^{T}=\bar{\varepsilon}_{i j}^{H}+\bar{\varepsilon}_{i j}^{M E}$, where $\bar{c}_{i j}=S_{i j k l} \sigma_{k l}^{a p p}$ is the Hookean strain and $S_{i j k l}$ is a compliance tensor. The total shear strain at given $\sigma$ is:

$\bar{\varepsilon}_{s h}^{T}=\bar{\varepsilon}_{s h}^{H}+\bar{\varepsilon}_{s h}^{M E}=\sigma / 2 C^{\prime}+\bar{\varepsilon}_{s h}^{M E}(\sigma)$,

where $\bar{\varepsilon}_{s h}^{M E}(\sigma)=\bar{\varepsilon}_{22}^{M E}(\sigma)-\bar{\varepsilon}_{33}^{M E}(\sigma)$ is the shear strain generated by embryos, $C^{\prime}=\left(C_{11}-C_{12}\right) / 2$ is a shear modulus, and $C_{11}$ and $C_{12}$ are the elastic moduli of the austenite (Supplemental materials). The shear strain $\bar{\varepsilon}_{s h}^{M E}$ is plotted as a function of $\sigma$ in Fig. 2a for several values of previous imposed plastic strain (hence, several dislocation densities). The figure shows that the strain amplification depends strongly on the density of dislocations, and is reversible and practically anhysteretic. This large increase in anhysteretic strain is a superelastic effect. ${ }^{14}$

Note that the often observed softening of pretransitional materials upon cooling is characterized by the effective isothermal modulus, ${ }^{23-25} C_{e}^{\prime}=\sigma / \bar{\varepsilon}_{s h}^{T}$. This softening is often considered as an intrinsic effect and attributed to incipient instability of the homogeneous austenite. However, softening in our case is due to an additional strain generated by increasing the equilibrium volume fraction of the embryos, $\omega$, upon cooling. A simple derivation gives,

$C_{e}^{\prime} / C^{\prime}=\left[1+\bar{\varepsilon}_{s h}^{M E} / \bar{\varepsilon}_{s h}^{H}\right]^{-1} \leq 1$.

It is known that $\bar{\varepsilon}_{s h}^{M E} \sim \varepsilon_{s h}^{0} \cdot \omega$, where $\varepsilon_{s h}^{0}$ is shear eigenstrain of the transformation. ${ }^{26}$ This embryo-induced strain is significant in most $\mathrm{fcc} \rightarrow \mathrm{bcc}$ transformations. Therefore, the effective modulus may decrease substantially if $\omega$ significantly increases upon cooling or loading (Fig. 2b).

When samples are cooled toward the $M_{s}$, the embryo size and, hence, $\omega$ increases monotonically from nanoscale to "infinity" (the sample size) (Fig. S2). It is noted that the $M_{s}$ temperature is a structure-sensitive value dependent on the nature and density of defects. This temperature is different from the congruent equilibrium temperature, $T_{o}$, that is an intrinsic parameter of the defect-free system determined by the equality of free energies of both stress-free phases at the same composition. The value of $M_{s}$ is determined as the instability temperature at which the system becomes unstable with respect to the growth of the coherent embryos to macroscopic dimension. The simulated change in the $M_{s}$ with prior plastic deformation is plotted in Fig. 3a.

The volume change of samples during cooling is plotted in Fig. $3 \mathrm{~b}$ for several values of the plastic strain. It is shown that the system volume increases during cooling toward $M_{s}$ and reversibly decreases on heating. This behavior is opposite to the conventional thermal expansion. Given the anhysteretic character of embryo growth, the net thermal expansion over this temperature range will reflect both embryo growth (shrinkage) and lattice expansion (contraction). The coefficient of thermal expansion will, hence, be reduced, and may vanish entirely. The latter behavior is the invar effect. ${ }^{18,19}$

In spite of the importance of the invar effect, its origin still remains controversial. Previous attempts to explain the invar effect by magnetic phenomena ${ }^{21,22}$ are problematic since the effect is also observed in nonmagnetic alloys, such as doped-NiTi alloys after aging. ${ }^{14}$ However, the invar effect does occur in pretransitional materials with low $M_{s,}$ e.g., the classic Fe-33 at $\% \mathrm{Ni}$ invar alloy has an $\mathrm{fcc} \rightarrow \mathrm{bcc}$ MT with $M_{s}$ as low as $\sim 223^{\circ} \mathrm{C} .{ }^{27}$ Besides, the hypothesis that pretransition embryo growth explains or at least contributes to the invar effect is supported by experiments showing that a severe cold-work treatment can significantly change the thermal expansion behaviors. ${ }^{19,28-31}$

A second interesting effect of increasing the equilibrium $\omega$ upon cooling is a softening of the effective modulus. This softening counteracts the usual stiffening (increase of $C^{\prime}$ ) on cooling. If these two thermal effects can cancel each other, the dependence of the elastic modulus on temperature vanishes. This is the so-called elinvar effect. ${ }^{19,32,33}$ Therefore, the dependence of the equilibrium $\omega$ on temperature can be an important source of both invar and elinvar effects.

Third, we consider the influence of magnetic fields on the equilibrium $\omega$. This behavior is particularly relevant when the product phase is ferromagnetic while the parent is not, which is the case for iron and many of its alloys. We may define an equivalent "magnetic stress" ${ }^{34}$ that makes the magnetic field producing the same effect as mechanical stress. For example, if a magnetic field is applied along the [0 $\overline{1} 1]$ direction, the equivalent "magnetic stress" on a tetragonal embryo is:

$\sigma^{\text {mag }}=H \cdot 2 \mu_{0} M_{0} /\left(\varepsilon_{c}-\varepsilon_{a}\right)$,

where $H$ is the magnitude of applied magnetic field, $\mu_{0}$ is vacuum permeability, $M_{0}$ is saturation magnetization of the product phase, $\varepsilon_{c}$ and $\varepsilon_{a}$ are diagonal components of its eigenstrain (Supplemental materials). Introducing a reference magnetic field, 


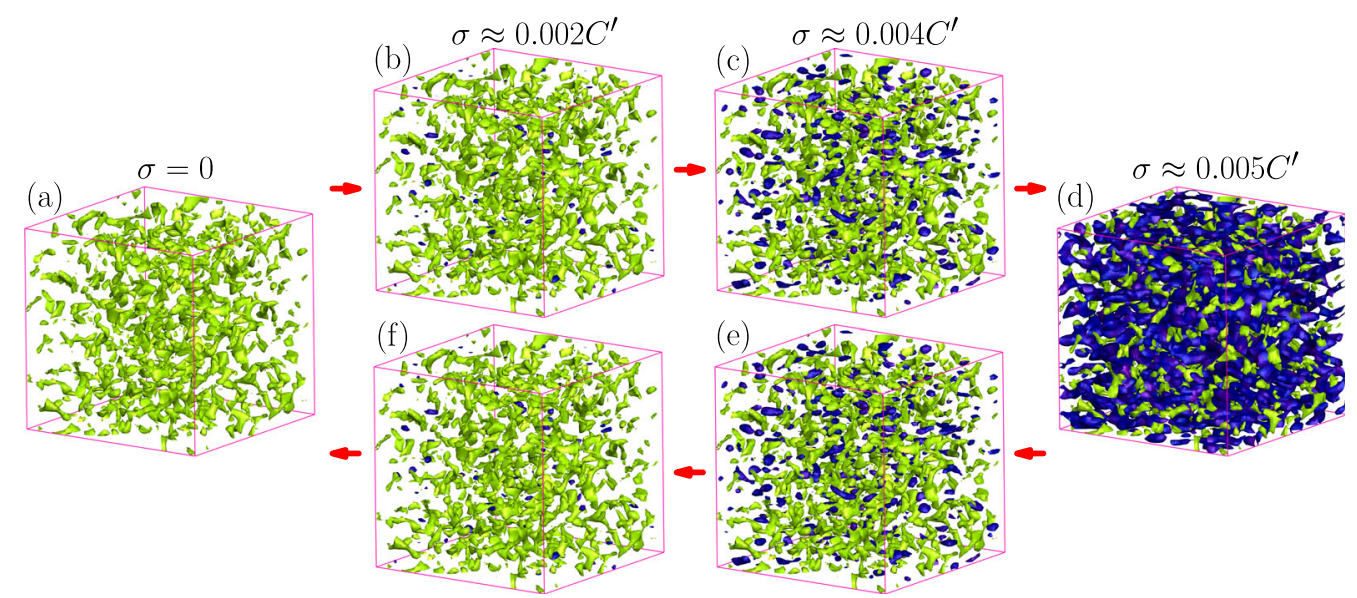

Fig. 4 The simulated evolution of the nanoembryonic martensitic structures (blue) in the pretransitional austenite with nanosize precipitates (green). The evolution is caused by quasi-static external stress cyclically applied at temperature $215 \mathrm{~K}$. The precipitates were obtained by simulating of early stages of decomposition of the pretransitional austenite. The volume fraction of precipitate phase, if in the thermodynamic equilibrium, is about $50 \%$. The assumed dilatation eigenstrain for precipitates is $\varepsilon_{p}=2 \%$. Red arrows indicate the sequence of the structures determined during the cycling of the applied shear stress

$H_{o}=C^{\prime}\left(\varepsilon_{c}-\varepsilon_{a}\right) / 2 \mu_{0} M_{0}$, estimated to be $2.5 \times 10^{9} \mathrm{~A} / \mathrm{m}\left(\sim 3.1 \times 10^{4}\right.$ kOe) for bcc Fe-31 at\% Ni, (Supplemental materials) we have the relation $H / H_{0}=\sigma / C^{\prime}$. This equivalency makes a plot of the magnetostrictive strain versus reduced magnetic field, $H / H_{0}$, to be identical to that of the strain versus reduced stress presented in Figs. 2a and 5, with $H / H_{0}$ replacing $\sigma / C^{\prime}$ as the abscissa.

The data shown in Fig. 2a suggest that the change of the equilibrium $\omega$ of a ferromagnetic product phase can produce a large and anhysteretic strain response to the applied magnetic field that in this case is a supermagnetostriction. Such a pretransitional material would be a member of a new class of magnetostrictors potentially competitive to the champion material, Terfenol-D. In principal, such alloys could be much cheaper than rare earth containing materials. Following the same line of reasoning, we could conclude that the nanoembryonic mixed state in a pretransitional material can also be multiferroic if the defect-rich pretransitional phase has different ferroic properties than the product phase.

Finally, we note that, like dislocations, nanoprecipitates with lattice misfit are also stress-generating defects that can produce thermoelastic equilibrium and stabilize embryos at $T>M_{s}$. When diffusion in pretransitional materials is allowed or unavoidable, the early stage of decomposition can produce a coherent nanodispersion of the precipitate phase. In our simulation, the misfit strain between the precipitate phase and the austenite is assumed to be dilatational (Supplemental materials). Figures 4 and 5 illustrate the formation and evolution of precipitate-induced embryos under changing applied shear stress [Eq. (1)] as well as the corresponding stress-strain curves. They are qualitatively identical to those obtained for dislocations, and thus, the static coherent precipitates may also result in a superelasticity and supermagnetostriction.

\section{DISCUSSION}

We employed computer simulations to study equilibrium states of pretransitional materials with stress-generating defects and their quasi-static responses to external stimuli. Here, the term pretransitional materials does not necessarily describe any heterogeneous microstructure in a precursor state since no morphology or relaxation phenomena has been discussed. It was shown that the localized stress-induced transformation around defects could produce nanosized embryos that are in a thermoelastic equilibrium with the matrix.

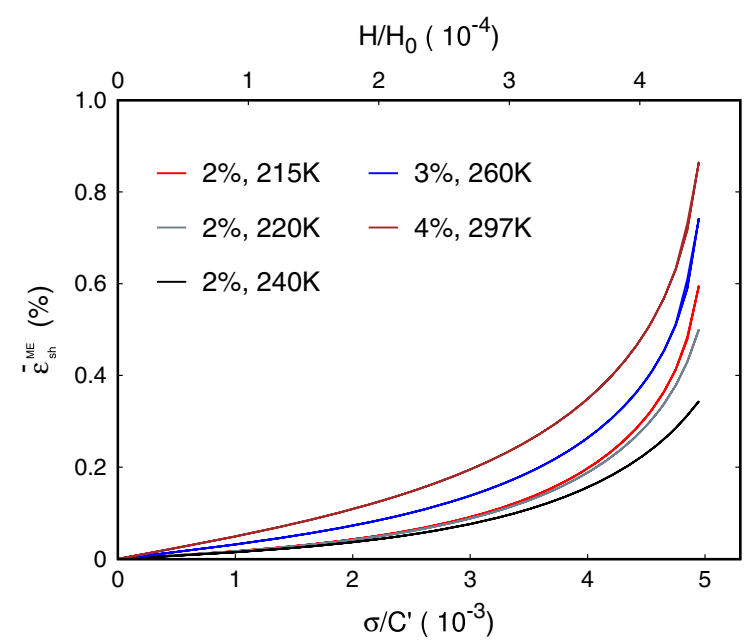

Fig. 5 Simulated shear strain amplifications versus cycling of applied stress and magnetic field ("magnetic stress") for pretransitional materials with $\sim 50 \%$ volume fraction of coherent nanoprecipitates. These curves describe the anhysteretic behavior of "samples" with different values of dilatational eigenstrains (lattice misfit between precipitate and austenite) at different temperatures (shown in the figure)

A similar problem of nanodispersive states has been previously studied, however, by substantially different computer models. In early models, ${ }^{35-38}$ only the local "contact" chemical interaction of point defects with martensite was considered, viz., defects were assumed to be impurity atoms only providing local spikes of the temperature of congruent transformation. These defects suppressing the transformation thus pinned the boundaries of nanosized particles formed by thermally activated nucleation and prevented the transformation of the system into a single phase state. In these models the typical size of particles was of the order of typical distance between defects. Later models also considered atomic point defects. ${ }^{39-41}$ However they took into account the interactions between the assumed dilatational point defects and martensite by introducing a local field effect to change the local stability and break the local symmetry and investigated the ratedependent responses.

We considered stress-generating dislocations and precipitates that are significantly more potent and complex defects than point 
defects. The size of embryos in our case is athermally determined by the thermoelastic equilibrium characterized by the defectembryo strain-induced interaction, which is, unlike in previous models, ${ }^{35-41}$ only weakly dependent on the distance between defects. That is, a quasi-static equilibration of embryos at each instantaneous value of external stimuli is not a kinetic but a thermodynamic process, and equilibrium embryos would not coarsen even if there was only a single defect. The gradual dependence of the equilibrium $\omega$ on temperature can be interpreted as a DPT. A jump of $\omega$ at the $M_{s}$ just describes a start of the conventional martensitic transformation upon cooling.

It was demonstrated that a shift of the thermoelastic equilibrium at $T>M_{s}$ upon changing external stimuli could automatically produce enhanced anhysteretic properties. For instance, such a shift upon cooling could produce a negative thermal expansion contributing to the invar effect if the martensite phase has a larger atomic volume (apparently the case for the generic FeNi invar alloys). The elinvar effect could be partly attributed to the elastic softening that is a direct result of the extra strain generated by increasing $\omega$ upon cooling, (Eq. (3)). However, our explanation does not exclude other contributions from different defect-based mechanisms, e.g., we do not exclude possible contributions to the invar effect by a cold-rolling induced texture of nanodomains, ${ }^{42,43}$ and to the elinvar effect by different modulus dependences on temperature for the parent and product phases. $^{42,43}$

We now consider the application of these concepts to known or potential examples having the described enhanced properties. An interesting example is the recently discovered "Gum Metal". ${ }^{19,29,44-47}$ The best of this material has nominal composition $\mathrm{Ti}_{23} \mathrm{Nb}_{0.7} \mathrm{Ta}_{2} \quad \mathrm{Zr}_{1.2} \mathrm{O}$ (at\%) and the bcc $(\beta)$ structure in the pretransitional state. After severe plastic deformation, the material shows a softening of the tensile modulus and a nonlinear anhysteretic elastic strain of $\sim 2.5 \%$. Together with this superelasticity, the material exhibits both invar and elinvar effects over a broad temperature range of about $100-500 \mathrm{~K} .{ }^{19}$ Moreover, alloys close to this composition are pretransitional since at low temperature they are unstable with respect to three separate martensitic transitions, to $a^{\prime}$ (hcp), $a^{\prime \prime}$ (orthorhombic), and $\omega$ (hexagonal). ${ }^{46}$ Recent in situ synchrotron studies of a closely related alloy, which is densely decorated with nanoprecipitates, document a reversible transformation to the $a^{\prime \prime}$ phase during $\langle 110\rangle$ tension that reaches a volume fraction of near $40 \%$. The alloy also has a superelastic behavior. ${ }^{47}$

Another known example is a superelasticity observed in NiTi after aging ${ }^{48}$ and plastic deformation ${ }^{14,49}$ and in the NiTi:Fe alloys after aging. ${ }^{50}$ The aged samples have a dense distribution of static precipitates and nanoembryos. The bcc NiTi alloys with near equiatomic compositions are pretransitional since at low temperature they form two different martensitic phases, the monoclinic B19' and rhombohedral R phases. ${ }^{14,49}$ The Fe-30 at\% Pd alloy with a tweed-like nanodispersion of single domain embryos is also a likely example. This alloy has about sevenfold softening of $C^{\prime}$ in the pretransitional state upon approaching the $M_{s}{ }^{23,24}$ This dramatic softening can be interpreted as superelasticity.

The nanoembryonic thermoelastic equilibrium at $T>M_{s}$ has even more interesting implications for ferromagnetic materials. This nanoembryonic thermoelastic equilibrium appears to explain two peaks of the anomalous magnetostriction of Fe-Ga alloys, ${ }^{51-}$ 55 whose magnetostriction reaches $\sim 400 \mathrm{ppm}$ in bcc solid solutions at $\sim 19$ at $\% \mathrm{Ga}$ and in $\mathrm{DO}_{3}$ ordered solutions at $\sim 28$ at $\%$ Ga. ${ }^{51-53}$ We attribute these giant magnetostrictions to the effect of elastic strain produced by nanoembryos that are stabilized by the local stresses of either coherent nanoprecipitates or static compositional nanoclusters. This conclusion is supported by: (i) magnetostriction in Fe-Ga alloys does not appear to be an intrinsic property since quenching from elevated temperature always produces a much higher magnetostriction than slow cooling $^{55}$; (ii) alloy compositions at both magnetostriction peaks practically coincide with the solubility limits of the bcc and the $\mathrm{DO}_{3}$ phases. These are the compositions at which the coherent stress-generating nanoprecipitates of, respectively, the $\mathrm{DO}_{3}$ and the $B_{2}$ phases start to form. In fact, HRTEM observations have confirmed the formation of coherent nanoprecipitates of the $\mathrm{DO}_{3}$ phase near 19 at\% Ga. ${ }^{52}$ (iii) Superelastic behavior has been reported at 23.8 at\% Ga, which is also the composition with enhanced magnetostriction observed. ${ }^{51,54}$ The coincidence of superelasticity and supermagnetostriction is expected because both effects can be caused by the same nanoembryonic mechanism.

There are other examples of enhanced magnetostriction in pretransitional alloys. One of them is bcc Fe-Co alloys near the fcc-bcc solubility limit, where the embryos have been observed near intermetallic nanoprecipitates, ${ }^{56}$ and magnetostriction can potentially reach $\sim 1000 \mathrm{ppm} .{ }^{57}$ In agreement with the nanoembryonic mechanism, plastic deformation of $\mathrm{Fe}_{x} \mathrm{Co}_{1-x}$ alloys near the solubility limit at $x=0.25$ increases the magnetostriction from 70 to 120 ppm. $^{56}$ Another probable example is both superelasticity and supermagnetostriction that have been observed in the Fe-Pd alloys with $M_{s} \sim 252 \mathrm{~K}^{58-60}$ The recently reported large magnetostriction in this alloy, ${ }^{60}$ though being attributed to the concept of strain glass, also follows from the nanoembryonic mechanism wherein the formation and evolution of embryos changes the first order MT to a diffuse (gradual) phase transition.

The nanoembryonic mechanism can also explain the origin of DPT and the property enhancement in ferroelectric pretransitional materials. For example, neutron diffraction studies of lead magnesium niobate and Ta-bearing strontium barium niobate ${ }^{61}$ revealed polar microregions within the paraelectric matrix that are readily identified as ferroelectric embryos formed by the ferroelectric transformation. The volume of these embryos gradually increases on cooling toward the Curie temperature (equivalent to the $M_{s}$ ) and causes a large increase in the dielectric permittivity.

We note that embryos can barrierlessly form in systems even when the stress generated by defects is not strong enough to induce a localized MT. In this case, no embryos could be observed in absence of applied field. However, even in this case, an application of the external field, which provides an additional driving force, may still make possible the reversible and anhysteretic formation of embryos and the corresponding superelasticity or supermagnetostriction. This effect can potentially be observed even in systems that have no MT at any temperature in absence of applied field. The latent instability in this case is transformed into a real one only by application of the external field.

In summary, we reported a nanoembryonic thermoelastic equilibrium of a nanoscale mixed state formed in highly defected pretransitional materials by the stress-induced transformation above the $M_{s}$. This equilibrium qualitatively differs from the conventional thermoelastic equilibrium of macroscopic crystals observed below the $M_{s}$. The nanoembryonic equilibrium can result in enhanced functional properties above the $M_{s,}$ and may partly explain the invar and elinvar effects. This equilibrium suggests a possible way for designing nonconventional functional materials with enhanced properties by using traditional treatments such as plastic deformation or aging.

\section{METHODS}

We used phase-field microelasticity (PFM) simulations to determine the morphology and properties of the embryos. The spatial distribution of all orientation variants in the simulations was characterized by their eigenstrain, $\varepsilon_{i j}^{o}(\mathbf{r})$, presented in terms of the long-range order (Iro) parameters $\eta_{p}(\mathbf{r})$ as $\varepsilon_{i j}^{o}(\mathbf{r})=\sum_{p=1}^{p=3} \varepsilon_{i j}^{B}(p) \eta_{p}(\mathbf{r})$, where $\eta_{p}(\mathbf{r})$ are density functions describing the probabilities that a point $\mathbf{r}$ is within a martensitic domain of the $p$ th type, the indices $p=1,2,3$ label three orientation 
variants of the tetragonal domains, and $\varepsilon_{i j}^{B}(p)$ are conventional Bain strains whose three tetragonality axes are along the $\langle 100\rangle$ directions of the $\mathrm{fcc}$ lattice (Supplemental materials). Any distribution of $\varepsilon_{i j}^{o}(\mathbf{r})$, determined by $\eta_{p}(\mathbf{r})$, generates an elastic strain, whose homogeneous part, $\bar{\varepsilon}_{i j}^{M E}$, is:

$\bar{\varepsilon}_{i j}^{M E}=\sum_{p=1}^{p=3} \varepsilon_{i j}^{o}(p)<\eta_{p}(\mathbf{r})>$,

where $\langle\ldots\rangle$ is an average over the sample volume, Hence, the volume fractions of the pth type of embryos, $\omega_{p}$, is $\omega_{p}=\left\langle\eta_{p}(\mathbf{r})\right\rangle$ and $\omega=\omega_{1}+\omega_{2}+$ $\omega_{3}$. The temporal and spatial evolutions of $\eta_{p}(\mathbf{r}, t)$ is governed by reduction of the free energy, which is described by the PFM kinetic equation (Supplemental materials). The free energy includes contributions of the stress-induced interaction between embryos, embryos and defects (sessile dislocations and static coherent precipitates of the third phase), and the interaction between embryos and the applied stress, magnetic and electric fields. "Sample preparations" were simulated as a result of plastic deformation caused by evolution of interacting dislocations including their spontaneous generation by randomly placed Frank-Read sources (Supplemental materials). ${ }^{62}$

\section{DATA AVAILABILITY}

The data that support the findings of this study are available from the corresponding author (wfrao@nuist.edu.cn or wfrao@hotmail.com) upon reasonable request.

\section{ACKNOWLEDGMENTS}

This work was partially supported by the National Natural Science Foundation of China (No. 11474167). The simulations were performed on NSCC-GZ.

\section{AUTHOR CONTRIBUTIONS}

W.F.R. and A.G.K. conceived and designed the project. W.F.R. and X.Y.C. performed the simulations. W.F.R., A.G.K., and X.Y.C. analyzed the results. W.F.R., A.G.K., X.Y.C., and J.W.M. wrote the manuscript.

\section{ADDITIONAL INFORMATION}

Supplementary information accompanies the paper on the npj Computational Materials website (https://doi.org/10.1038/s41524-018-0114-7).

Competing interests: The authors declare no competing interests.

Publisher's note: Springer Nature remains neutral with regard to jurisdictional claims in published maps and institutional affiliations.

\section{REFERENCES}

1. Oikawa, K. et al. Magnetic and martensitic phase transitions in ferromagnetic $\mathrm{Ni}-\mathrm{Ga}-\mathrm{Fe}$ shape memory alloys. Appl. Phys. Lett. 81, 5201-5203 (2002).

2. Kakeshita, T., Saburi, T., Kind, K. \& Endo, S. Martensitic transformations in some ferrous and non-ferrous alloys under magnetic field and hydrostatic pressure. Phase Transit. 70, 65-113 (1999).

3. Sozinov, A., Likhachev, A. A., Lanska, N. \& Ullakko, K. Giant magnetic-field-induced strain in NiMnGa seven-layered martensitic phase. Appl. Phys. Lett. 80, 1746-1748 (2002).

4. Tanaka, Y. et al. Ferrous polycrystalline shape-memory alloy showing huge superelasticity. Science 327, 1488-1490 (2010).

5. Levitas, V. I. High-pressure mechanochemistry: conceptual multiscale theory and interpretation of experiments. Phys. Rev. B 70, 184118 (2004).

6. Javanbakht, M. \& Levitas, V. I. Phase field simulations of plastic strain-induced phase transformations under high pressure and large shear. Phys. Rev. B 94, 214104 (2016).

7. Javanbakht, M. \& Levitas, V. I. Nanoscale mechanisms for high-pressure mechanochemistry: a phase field study. J. Mater. Sci. 53, 13343-13363 (2018).

8. Olson, G. B. \& Cohen, M. A general mechanism of martensitic nucleation: Part II. FCC->BCC and other martensitic transformations. Metall. Trans. A 7, 1905-1914 (1976).

9. Olson, G. B. \& Roytburd, A. L. Atribute to Morris Cohen. in Martensite (eds Olson, G. B. \& Owen, W. S.) (ASM International Materials Park OH, 1992).

10. Olson, G. B. \& Cohen, M. Dislocation theory of martensitic transformations. Dislocations in Solids (North-Holland, Elsevier Science Publisers B.V., 1986).
11. Kurdyumov, G. \& Khandros, L. On the "thermoelastic" equilibrium on martensitic transformations. Dokl. Akad. Nauk SSSR 66, 211-214 (1949).

12. Roytburd, A. L. Nucleation on dislocations in martensitic transformations. Sov. Phys. Dokl. 26, 92-94 (1981).

13. Slutsker, J. \& Roytburd, A. L. Control of intrinsic instability of superelastic deformation. Int. J. Plast. 18, 1561-1581 (2002).

14. Otsuka, K. \& Ren, X. Physical metallurgy of Ti-Ni-based shape memory alloys. Prog. Mater. Sci. 50, 511-678 (2005).

15. Khachaturyan, A. G. \& Viehland, D. Structurally heterogeneous model of extrinsic magnetostriction for Fe-Ga and similar magnetic alloys: Part I. decomposition and confined displacive transformation. Metall. Mater. Trans. A 38 A, 2308-2316 (2007).

16. Khachaturyan, A. G. \& Viehland, D. Structurally heterogeneous model of extrinsic magnetostriction for Fe-Ga and similar magnetic alloys: Part II. Giant magnetostriction and elastic softening. Metall. Mater. Trans. A 38, 2317-2328 (2007).

17. Isupov, V. A. Some problems of diffuse ferroelectric phase transitions. Ferroelectrics 90, 113-118 (1989).

18. Guillaume, C. E. The anomaly of the nickel-steels. Proc. Phys. Soc. Lond. 32, 374-404 (1919).

19. Saito, T. et al. Multifunctional alloys obtained via a dislocation-free plastic deformation mechanism. Science 300, 464-467 (2003).

20. Eshelby, J. D. The determination of the elastic field of an ellipsoidal inclusion, and related problems. Proc. R. Soc. A Math. Phys. Eng. Sci. 241, 376-396 (1957).

21. Weiss, R. J. The origin of the "Invar" effect. Proc. Phys. Soc. 82, 281-288 (1963).

22. Van Schilfgaarde, M., Abrikosov, I. A. \& Johansson, B. Origin of the invar effect in iron-nickel alloys. Nature 400, 46-49 (1999).

23. Mut, S., Oshima, R., Fujita, F. E. \& Muto, S. Elastic softening and elastic strain energy consideration in the FCC-FCT transformation of Fe-Pd alloys. Acta Metall. 38, 685-694 (1990).

24. Chopra, H. D. \& Wuttig, M. Precursor shear elastic pseudo-isotropy in near second order martensitic phase transitions. J. Phys. IV Colloq. C8, C8/157 (1995).

25. Wuttig, M., Dai, L. \& Cullen, J. Elasticity and magnetoelasticity of Fe-Ga solid solutions. Appl. Phys. Lett. 80, 1135-1137 (2002).

26. Khachaturyan, A. G. Theory of Structural Transformations in Solids. (John Wiley \& Sons, Inc., New York, 1983).

27. Kaufman, L. \& Cohen, M. The martensitic transformation in the iron-nickel system. JOM 8, 1393-1401 (1956)

28. Kainuma, R., Wang, J. J., Omori, T., Sutou, Y. \& Ishida, K. Invar-type effect induced by cold-rolling deformation in shape memory alloys. Appl. Phys. Lett. 80, 4348-4350 (2002).

29. Nakai, M. et al. Anomalous thermal expansion of cold-rolled Ti-Nb-Ta-Zr alloy. Mater. Trans. 50, 423-426 (2009).

30. Hao, Y. L. et al. Superelasticity and tunable thermal expansion across a wide temperature range. J. Mater. Sci. Technol. 32, 705-709 (2016).

31. Ahadi, A., Matsushita, Y., Sawaguchi, T., Sun, Q. P. \& Tsuchiya, K. Origin of zero and negative thermal expansion in severely deformed superelastic NiTi alloy. Acta Mater. 124, 79-92 (2017).

32. Cui, J. \& Ren, X. Elinvar effect in Co-doped TiNi strain glass alloys. Appl. Phys. Lett. 105, 061904 (2014).

33. Zhang, L., Wang, D., Ren, X. \& Wang, Y. A new mechanism for low and temperature-independent elastic modulus. Sci. Rep. 5, 11477 (2015).

34. Rao, W. F. \& Khachaturyan, A. G. Superfunctionalities in nanodispersive precipitation-hardened alloys. Phys. Rev. Lett. 109, 115704 (2012).

35. Kartha, S., Castn, T., Krumhansl, J. A. \& Sethna, J. P. Spin-glass nature of tweed precursors in martensitic transformations. Phys. Rev. Lett. 67, 3630-3633 (1991).

36. Kartha, S., Krumhansl, J., Sethna, J. \& Wickham, L. Disorder-driven pretransitional tweed in martensitic transformations. Phys. Rev. B 52, 803 (1995).

37. Semenovskaya, S. \& Khachaturyan, A. G. Coherent structural transformations in random crystalline systems. Acta Mater. 45, 4367-4384 (1997).

38. Semenovskaya, S. \& Khachaturyan, A. G. Development of ferroelectric mixed states in a random field of static defects. J. Appl. Phys. 83, 5125-5136 (1998).

39. Wang, D., Wang, Y., Zhang, Z. \& Ren, X. Modeling abnormal strain states in ferroelastic systems: the role of point defects. Phys. Rev. Lett. 105, 205702 (2010).

40. Wang, D. et al. Superelasticity of slim hysteresis over a wide temperature range by nanodomains of martensite. Acta Mater. 66, 349-359 (2014).

41. Wang, D. et al. Defect strength and strain glass state in ferroelastic systems. J. Alloy. Compd. 661, 100-109 (2016).

42. Ji, Y. et al. Ferroic glasses. npj Comput. Mater. 3, 43 (2017).

43. Wang, Y. et al. Strain glass transition in a multifunctional $\beta$-type Ti alloy. Sci. Rep. 4, 3995 (2014).

44. Talling, R. J., Dashwood, R. J., Jackson, M. \& Dye, D. On the mechanism of superelasticity in gum metal. Acta Mater. 57, 1188-1198 (2009).

45. Morris, J. W. et al. Anomalous transformation-induced deformation in $\left\langle\begin{array}{lll}1 & 1 & 0\end{array}\right\rangle$ textured gum metal. Acta Mater. 58, 3271-3280 (2010). 
46. Lazar, P. et al. Temperature-induced martensitic phase transitions in gum-metal approximants: first-principles investigations for $\mathrm{Ti}_{3} \mathrm{Nb}$. Phys. Rev. B 84, 054202 (2011).

47. Liu, J.-P. et al. New intrinsic mechanism on gum-like superelasticity of multifunctional alloys. Sci. Rep. 3, 2156 (2013).

48. Sehitoglu, H. et al. Shape memory and pseudoelastic behavior of $51.5 \% \mathrm{Ni}-\mathrm{Ti}$ single crystals in solutionized and overaged state. Acta Mater. 49, 3609-3620 (2001).

49. Liang, Q. et al. Novel B19' strain glass with large recoverable strain. Phys. Rev. Mater. 1, 033608 (2017).

50. Zhang, J. et al. Stress-induced strain glass to martensite (R) transition in a $\mathrm{Ti}_{50} \mathrm{Ni}_{44.5} \mathrm{Fe}_{5.5}$ alloy. Phys. Rev. B 83, 174204 (2011).

51. Ikeda, O., Kainuma, R., Ohnuma, I., Fukamichi, K. \& Ishida, K. Phase equilibria and stability of ordered b.c.c. phases in the Fe-rich portion of the Fe-Ga system. J. Alloy. Compd. 347, 198-205 (2002).

52. Bhattacharyya, S. et al. Nanodispersed $\mathrm{DO}_{3}$-phase nanostructures observed in magnetostrictive Fe-19\% Ga Galfenol alloys. Phys. Rev. B 77, 104107 (2008).

53. Xing, Q., Du, Y., McQueeney, R. J. \& Lograsso, T. A. Structural investigations of Fe-Ga alloys: phase relations and magnetostrictive behavior. Acta Mater. 56 4536-4546 (2008).

54. Yasuda, H. Y., Oda, Y., Aoki, M., Fukushima, K. \& Umakoshi, Y. Multimode pseudoelasticity in Fe-23.8 at\% Ga single crystals with $\mathrm{DO}_{3}$ structure. Intermetallics 16, 1298-1304 (2008).

55. Clark, A. E., Wun-Fogle, M., Restorff, J. B., Lograsso, T. A. \& Cullen, J. R. Effect of quenching on the magnetostriction on $\mathrm{Fe}_{1-x} \mathrm{Ga}_{x}(0.13<x<0.21)$. IEEE Trans. Magn. 37, 2678-2680 (2001).

56. Yamaura, S., Nakajima, T., Satoh, T., Ebata, T. \& Furuya, Y. Magnetostriction of heavily deformed Fe-Co binary alloys prepared by forging and cold rolling. Mater. Sci. Eng. B 193, 121-129 (2015).
57. Hunter, D. et al. Giant magnetostriction in annealed $\mathrm{Co}_{1-x} \mathrm{Fe}_{x}$ thin-films. Nat Commun. 2, 518 (2011)

58. James, R. D. \& Wuttig, M. Magnetostriction of martensite. Philos. Mag. A 77, 1273-1299 (1998).

59. Steiner, J., Lisfi, A., Kakeshita, T., Fukuda, T. \& Wuttig, M. Unique magnetostriction of $\mathrm{Fe}_{68.8} \mathrm{Pd}_{31.2}$ attributable to twinning. Sci. Rep. 6, 34259 (2016).

60. Ren, S. et al. Low-field-triggered large magnetostriction in iron-palladium strain glass alloys. Phys. Rev. Lett. 119, 125701 (2017).

61. Tsurumi, T., Soejima, K., Kamiya, T. \& Daimon, M. Mechanism of diffuse phase transition in relaxor ferroelectrics. Jpn. J. Appl. Phys. 33, 1959-1964 (1994).

62. Wang, Y. U., Jin, Y. M., Cuitiño, A. M. \& Khachaturyan, A. G. Nanoscale phase field microelasticity theory of dislocations: model and 3D simulations. Acta Mater. 49, 1847-1857 (2001).

Open Access This article is licensed under a Creative Commons Attribution 4.0 International License, which permits use, sharing, adaptation, distribution and reproduction in any medium or format, as long as you give appropriate credit to the original author(s) and the source, provide a link to the Creative Commons license, and indicate if changes were made. The images or other third party material in this article are included in the article's Creative Commons license, unless indicated otherwise in a credit line to the material. If material is not included in the article's Creative Commons license and your intended use is not permitted by statutory regulation or exceeds the permitted use, you will need to obtain permission directly from the copyright holder. To view a copy of this license, visit http://creativecommons. org/licenses/by/4.0/.

(c) The Author(s) 2018 